Journal for ImmunoTherapy of Cancer

\section{Mechanisms underlying low-clinical responses to PD-1/PD-L1 blocking antibodies in immunotherapy of cancer: a key role of exosomal PD-L1}

To cite: Yin Z, Yu M, Ma T, et al. Mechanisms underlying low-clinical responses to PD-1/ PD-L1 blocking antibodies in immunotherapy of cancer: a key role of exosomal PD-L1. Journal for ImmunoTherapy of Cancer 2021;9:e001698. doi:10.1136/ jitc-2020-001698

ZY and MY contributed equally. Accepted 16 December 2020

Check for updates

(C) Author(s) (or their employer(s)) 2021. Re-use permitted under CC BY-NC. No commercial re-use. See rights and permissions. Published by BMJ.

${ }^{1}$ Department of General Surgery, Guangdong Provincial People's Hospital, Guangdong Academy of Medical Science, Guangzhou, China

${ }^{2}$ Department of Obstetrics and Gynecology, Sun Yat Sen Memorial Hospital, Sun Yat sen University, Guangzhou, China ${ }^{3}$ Department of Medical Genetics, School of Medicine, Bam University of Medical Sciences, Bam, Iran

${ }^{4}$ Department of Medical Biotechnology, School of Medicine, Alborz University of Medical Sciences, Karaj, Iran

Correspondence to

Dr Zi Yin; yinzi@gdph.org.cn

Dr Sheng Chen; chenshenglucky@yeah.net

\section{ABSTRACT}

Exosomes, as the main group of extracellular vesicles, are biologically active lipid-bilayer vesicles that are naturally released from different types of normal or tumor cells. These vesicles play an important role in intercellular communication and influence the extracellular environment and the immune system. Emerging evidence demonstrates that cancer-derived exosomes are enriched in immunosuppressive proteins, such as the programmed death-ligand 1 (PD-L1). PD-L1 and its receptor programmed cell death protein $1(\mathrm{PD}-1)$ are the key immune checkpoint molecules that promote tumor progression via negative regulation of immune responses. PDL-1 is highly expressed on the surface of tumor cells and binds to PD-1 on the surface of activated T cells, leading to suppression of T cells, which consequently enables cancer cells to escape antitumor immunity. Currently, there are several Food and Drug Administrationapproved monoclonal antibodies blocking PD-1/PD-L1 interaction, which are clinically used for cancer treatment. However, despite impressive treatment outcomes, some patients show poor response to PD-1/PD-L1 blockade. Of note, tumor-derived exosomes containing PD-L1 can recapitulate the effect of cell-surface PD-L1. There is evidence that reveals a significant association between levels of circulating exosomal PD-L1 and rate of response to anti-PD-1/PD-L1 antibody therapy. The present article reviews the role of exosomal PDL- 1 in the therapeutic resistance to anti-PD-1/PD-L1 treatment. Importantly, it is suggested that the removal of exosomal PDL-1 could serve as a therapeutic adjuvant for enhancing the efficacy of anti-PD-1/PD-L1 therapy in patients with cancer.

\section{PD-1/PD-L1 IMMUNE CHECKPOINT PATHWAY AS A DOUBLE-EDGED SWORD}

Immune checkpoints are cell surface regulatory receptors, located mainly, but not exclusively, on $\mathrm{T}$ lymphocytes. After recognizing cognate ligands on the antigen-presenting cells or the target cells, these receptors act as $\mathrm{T}$ cell receptor (TCR) cosignaling partners that deliver either stimulatory or inhibitory signals to regulate the lymphocyte activation. ${ }^{1}$
Programmed cell death protein 1 (PD-1) is a key immune checkpoint molecule known as one of the major inhibitory coreceptors expressed in the activated $\mathrm{T}$ cells. ${ }^{2}$ Under normal physiology, immune checkpoints, such as PD-1, are critical for maintaining self-tolerance, preventing autoimmunity, and controlling T-cell responses within a desired physiological range to protect tissues from excessive inflammatory reactions. However, in cancerous conditions, these regulatory proteins can allow tumor cells to protect themselves from the antitumor T-cell responses, causing the so-called tumor immune evasion. ${ }^{1}$

PD-1 acts through interaction with PD-L1 that is a highly expressed ligand in nonlymphoid tissues in response to inflammatory cytokines such as interferon-gamma (IFN- $\gamma$ ) produced by cytotoxic T Cells. ${ }^{23}$ On engagement with PD-L1, PD-1 transmits a negative costimulatory signal in $\mathrm{T}$ cells through the recruitment of Src homology 2 domain containing phosphatases $1 / 2$ (SHP1/2) that dephosphorylates downstream TCRmediated signaling elements and thereby inhibits T cell proliferation, cytokine production and release, and cytotoxicity. ${ }^{4}$ Therefore, the PD-1/PD-L1 regulatory system is induced by immune responses and then through a negative feedback loop attenuates T-cell responses and minimizes tissue damage.

On tumor antigen recognition by $\mathrm{T}$ cells, the released IFN- $\gamma$ triggers tumor cells to express PD-L1 that binds coinhibitory receptor molecule PD-1 on cytotoxic T cells within the tumor microenvironment (TME). This allows the specific inhibition of tumor recognition by $\mathrm{T}$ cells, resulting in attenuating antitumor immune responses and promoting tumor growth. IFN- $\gamma$ induces the expression of PD-L1 through activating signaling pathways downstream of the type II interferon 
Table 1 FDA-approved monoclonal antibodies blocking immune checkpoints in human cancer

\begin{tabular}{llll} 
Target & Therapeutic antibody & Tumor type & FDA approval year \\
\hline CTLA4 & Ipilimumab & Melanoma, renal cell carcinoma, metastatic colorectal cancer & 2011 \\
PD-1 & Pembrolizumab & Melanoma, non-small cell lung cancer, renal cell carcinoma, urothelial & 2014 \\
& & $\begin{array}{l}\text { bladder cancer, Hodgkin's lymphoma, head and neck cancer, Merkel } \\
\text { cell carcinoma, microsatellite instability-high cancer, gastric cancer, } \\
\end{array}$ & hepatocellular carcinoma, cervical cancer, primary mediastinal large \\
& & B-cell lymphoma
\end{tabular}

Nivolumab Melanoma, non-small cell lung cancer, renal cell carcinoma, urothelial 2014 bladder cancer, Hodgkin's lymphoma, head and neck cancer, colorectal cancer, hepatocellular carcinoma, small cell lung cancer

Cemiplimab

Cutaneous squamous-cell carcinoma

2018

\begin{tabular}{llll} 
PD-L1 Atezolizumab & Non-small cell lung cancer, urothelial bladder cancer, small cell lung & 2016 \\
& cancer, breast cancer & 2017 \\
Avelumab & Merkel cell carcinoma, urothelial bladder cancer & 2017 \\
Durvalumab & Non-small cell lung cancer, urothelial bladder cancer & \\
\hline
\end{tabular}

Data have been acquired from "Timeline of Anti-PD-1/L1 Antibody Approvals by the FDA." Available in online (https://www.cancerresearch. org/scientists/immuno-oncology-landscape/pd-1-pd-I1-landscape). IFN- $\gamma$, interferon-gamma; PD-1, programmed cell death protein 1; PD-L1, programmed death-ligand 1.

receptor, including Janus kinase and signal transducer and activators of transcription, leading to binding of the IRF1 transcription factor to the PD-L1 gene promoter. $^{5}$ Moreover, oncogenic pathways, including MAPK (RAS/ $\mathrm{RAF} / \mathrm{MEK} / \mathrm{ERK}$ ) and PI3K/Akt/mTOR, are also known to mediate the induction of PD-L1 expression through activating c-JUN that, as the component of AP-1 transcription factor, binds to the enhancer element on PD-L1 gene and augments the transcription signal in tumor cells. ${ }^{6}$

\section{THERAPEUTIC EFFECTS OF PD-1/PD-L1 BLOCKING ANTIBODIES}

Immune checkpoint blockade therapies are now approved by US Food and Drug Administration (USFDA) for the treatment of a wide range of cancer types, with approval likely for additional indications in the near future. Among negative immune checkpoint molecules, the blockade of PD-1 and its major ligand, PD-L1, provides one of the most successful immunotherapies by intensifying T cell immunity against cancer cells. Since the 2011 FDA approval of the first immune checkpoint inhibitor, ipilimumab (anti-CTLA4), for the treatment of metastatic melanoma, six monoclonal antibodies targeting the PD-1 (pembrolizumab, nivolumab, and cemiplimab) and PD-L1 (atezolizumab, durvalumab, and avelumab), have been approved for the treatment of various cancer types (table 1). ${ }^{1}$

An important rationale respecting the development of anti-PD-1/PD-L1 drugs for cancer treatment emerged from the key findings that showed PD-1 is over-expressed on tumor-infiltrating lymphocytes (TILs), ${ }^{78}$ while PD-1 ligand is highly upregulated in most of the human cancer cells. ${ }^{9}$ This was validated through many preclinical studies that indicated mAbs blockade of PD-1 or PD-L1 significantly improved antitumor immunity. ${ }^{9-11}$ Anti-PD-1/ PD-L1 blocking mAbs have heightened tumor selectivity and decreased toxicity as well as a much broader spectrum of antitumor activity when compared with antiCTLA-4 mAbs. There is evidence that clinical responses to immune checkpoint inhibitors might be correlated with immune-related adverse events (irAEs). Overall, drug-induced irAEs are more likely to be experienced in patients treated with anti-CTLA- $4(60 \%-85 \%)$ than antiPD-1 (16\%-37\%) or anti-PD-L1 $(12 \%-24 \%){ }^{12-14}$

Pembrolizumab, the first mAbs against PD-1, achieved its first global approval for patients with unresectable or metastatic melanoma by US-FDA. ${ }^{15}$ Afterward, its use was extended to non-small cell lung cancer (NSCLC) ${ }^{16}$ head and neck squamous cell carcinoma (HNSCC) ${ }^{17}$ cervical carcinoma $^{18}$ and metastatic urothelial carcinoma $^{18}$ among others in a list that continues to grow. Anti-PD-1 antibodies can block the PD-1 signaling pathway, thus preventing PD-1-mediated attenuation of TCR signaling, which promotes reinvigoration of exhausted PD- $1^{+} \mathrm{CD} 8^{+} \mathrm{T}$ cells, resulting in immune tumor rejection. ${ }^{19-22}$ Although the precise molecular and cellular events mediating enhancement of antitumor immunity by PD-1 blockade are not fully understood, tumor neoantigen-specific $\mathrm{CD}^{+}$ $\mathrm{T}$ cells appear to be the major T-cell population mediating anti-PD-1 responses. ${ }^{23}$ Apart from restoring T-cell activity through modulation of TCR signaling, blockade of the PD-1 signaling axis is also able to reverse the associated metabolic reprogramming to an extent which in part mediates the reinvigoration of tumor antigen-specific $\mathrm{T}$ cells. $^{2425}$

Alongside direct inhibition of PD-1, antibodies targeting PD-L1 are also sufficient to reverse the negative immune regulation and, thereby, reinvigorate the host antitumor immunity. The current FDA-approved mAbs targeting PD-L1 are known to be efficient for treating several cancer types, including NSCLC, small cell lung 
cancer, urothelial bladder cancer, breast cancer, and Merkel cell carcinoma. ${ }^{1}$ In the light of the dominance in PD-L1 expression, blockade of PD-L1 can reiterate the impact of PD-1 blockade. PD-L1 expression is primarily triggered by Th1 cytokines, such as IFN- $\gamma$. This can in part describe the effectiveness of PD-L1 blockade since Th1skewed responses would be more desirable for inducing antitumor immunity ${ }^{26}$ By contrast with PD-1 blockade, anti-PD-L1 antibodies may also achieve part of their efficacy from antibody-dependent cellular cytotoxicity. This claim emerged from an in vivo study that shows Fc receptor binding is crucial for the effectiveness of antiPD-L1, but not anti-PD-1, antibody therapy-promoted tumor regression in mice bearing tumor. ${ }^{27}$ Notably, these findings suggest that anti-PD-1 and anti-PD-L1 antibodies have distinct biological activities and act, at least in part, through different mechanisms.

\section{FACTORS MODULATING RESPONSE TO PD-1/PD-L1 INHIBITORS}

Despite the above-mentioned advancements in clinical practice, some patients and cancer types show therapeutic resistance or relatively low-response rate to PD-1/ PD-L1 inhibitors. ${ }^{28-30}$ The basis of differential therapeutic success between patients and between cancers is not completely understood. However, several factors, such as the TME, the tumor genomics, and systemic factors such as exosomes have been suggested for explaining potential mechanisms of the response and resistance to PD-1/ PD-L1 inhibitors.

Of TME-related factors, PD-L1 expression status and pre-existing TILs have been widely studied..$^{20}$ Although the conclusions from various clinical trials are not consistent, a significant positive but not absolute correlation between PD-L1 expression in the TME and responsiveness to anti-PD-1/anti-PD-L1 therapy has been generally found. ${ }^{32}{ }^{33}$ Across all tumor types, patients with PD-L1negative tumors respond to anti-PD-1/PD-L1 therapy in 0\%-17\%, while those with PD-L1-positive tumors exhibit a response rate ranging from $44 \%$ to $100 \% .{ }^{34}$ Besides, TILs are the other cell types affecting the tumor immune microenvironment. The density and phenotype of preexisting TILs within a TME can impact the efficacy of antiPD-1/PDL1 therapy. ${ }^{35}$ The results from a model of tumor immune microenvironment that consists of TILs and PD-L1 expression status indicate that patients with cancer with PD-L1 ${ }^{+} \mathrm{TIL}^{+}$tumors possess an effective immune response to PD-1/PD-L1 blockade therapy. ${ }^{36}$ In patients with NSCLC, pretherapy levels of the intratumoral population of $\mathrm{CD}^{+}$cytotoxic $\mathrm{T}$ cells with the highest PD-1 expression have shown a positive correlation with antiPD-1 response. ${ }^{37}$ Moreover, the ratio of memory-like to exhausted TILs, as two major intratumoral $\mathrm{CD}^{+} \mathrm{T}$ cell phenotypes, has been indicated to be strongly correlated with increased survival and response in a cohort of patients with melanoma treated with anti-PD-1. ${ }^{38}$

Another studied factor is the tumor mutation burden (TMB). The primary targets of many tumor immune responses are tumor-specific neoantigen peptides that arise from somatic mutations in tumor genomes. The number of non-synonymous single nucleotide variants in a tumor, referred to as TMB, can affect the odds of generating immunogenic peptides and thereby influence response to immune checkpoint blockade in patients. ${ }^{25}{ }^{39-44}$ Of note, a higher TMB favors positive response to PD-1/PD-L1 blockade in a variety of tumor types including NSCLC, ${ }^{23}{ }^{45-48}$ small cell lung cancer, ${ }^{48}$ urothelial carcinoma $^{49}$ metastatic melanoma, ${ }^{50}$ and HNSCC. ${ }^{51-53}$ Moreover, data from a meta-analysis across 27 tumor types show a positive correlation between average response rate and TMB. ${ }^{54}$ These findings make clear that there is a significant pan-cancer association between TMB and response to PD-1/PD-L1 blockade by the tumor type. Further supporting the relationship between TMB and high sensitivity to PD-1/PD-L1 blockade is the observation that microsatellite instability (MSI)-high/mismatch repair deficiency (MMRd) associates with satisfactory treatment effects in patients with multiple cancer receiving anti-PD-1/PD-L1 therapy. ${ }^{55}$ Tumors with high MSI generate many neopeptides owing to the hypermutated phenotype. MSI-positive tumors are a specific type of high TMB tumors, with MMRd generating a high mutational load. Notably, MMRd derives several insertion and deletion mutations resulting in frameshifts producing neoantigens that may be more immunogenic due to their higher sequence divergence from self-peptides. ${ }^{5-57}$ Taken together, these findings reveal that MMRd is correlated with enhanced response to PD-1/PD-L1 blockade because of increased TMB.

Besides, systemic factors are also found to influence the therapeutic efficacy of PD-1/PD-L1 inhibitors. Among them, peripheral blood parameters including neutrophil/lymphocyte ratio, total lymphocyte and monocyte count, relative eosinophil count, T cell clonality, PD-L1 $1^{\text {high }}$ circulating tumor cells, and circulating PD-L1 have been reported to be significantly correlated with PD-1/PD-L1 blockade response in multiple studies of anti-PD-1/PD-L1 therapy across a wide range of cancer types. ${ }^{58}$ Exosomal PD-L1 is another systemic factor that has recently attracted extensive attention. Tumor cells release PDL1containing exosomes that have been known to be in part responsible for resistance to anti-PD-1/PDL-1 therapy. A growing body of evidence indicated that exosomal PDL-1 affects the TME and antitumor immunity. In the present review article, we seek impacts of exosomal PD-L1 on clinical outcomes with PD-1/PDL-1 blockade therapy and suggest potential combination therapies to overcome the resistance.

\section{ACTIVITY OF EXOSOMAL PD-L1}

Exosomes, as the main group of secreted small extracellular vesicles, are biologically active lipid-bilayer vesicles with a size around $30-100 \mathrm{~nm}$ that are naturally produced and released by different types of normal and tumor cells. ${ }^{5960}$ These vesicles play an important role in 
intercellular communication and influence the extracellular environment and the immune system responses. ${ }^{5961}$ Exosomes are secreted into extracellular space through endosomal pathways and transport various bioactive molecules (cargo) to the target cells. The composition of exosomal cargo is very diverse and includes a wide range of immunosuppressive and immunostimulatory proteins, chemokines, cytokines, cellular receptors, lipids, as well as different nucleic acids such as micro-RNAs and circular RNAs. ${ }^{62-64}$

Tumor cells can actively produce large levels of exosomes enriched in cancer-promoting cellular contents, such as immunosuppressive proteins like PD-L1, mRNAs, and micro-RNAs, that participate in cancer development and metastasis, through dysregulating antitumor or protumor immunity responses and promoting drug resistance.$^{65-67}$ Of note, tumor-secreted exosomes contain PD-L1 presented both on the surface and within exosome particles. ESCRTs (endosomal sorting complex required for transport) are involved in the packaging of biomolecules into exosome, and nSMase2 (neutral sphingomyelinase 2) and Rab proteins regulate exosome secretion. ${ }^{68}$ ESCRT, Rab27a, and nSMase2 have been identified to participate in the packaging and secretion of exosomal PD-L1 ${ }^{69}$ Exosomes can transport PD-L1 to other cells with low or no PD-L1 expression, with the potential to bind to PD-1. ${ }^{70}$

The plasma/serum levels of PD-L1 expressed on exosomes, but not soluble PD-L1, are found to associate with disease progression and clinicopathological features in patients with cancer, such as HNSCC ${ }^{71}$ and NSCLC. ${ }^{72}$ Of note, major histocompatibility complex (MHC) molecules expressed on exosomes can play an essential role in exosomal PD-L1-mediated tumor promotion. Indeed, molecular interaction of exosomal MHC I with TCR enhances the inhibitory effect of exosomal PD-L1 to T cells. These might be perfectly justifiable reasons for the higher immunosuppressive impact of exosomal PD-L1 than the soluble form. ${ }^{71} 72$

Exosomal PD-L1 can recapitulate the effect of cellsurface PD-L1; it similarly provokes tumor progression mainly through enabling cancer cells to escape antitumor immunity via inhibiting $\mathrm{T}$ cell activation (figure 1). Generally, B cell presentation of antigen to $\mathrm{T}$ cells will activate T cells. An in vitro setting of PD-L1 negative Raji B cell and PD-1 expressing Jurkat T cell revealed that exosomal PD-L1 can suppress T cell activation. ${ }^{73}$ The immunosuppressive effect of tumor-derived exosomal PD-L1 has been widely confirmed by other investigations. Malignant glioma cells have been found to generate exosomes containing PD-L1 that involves in tumor progression. Exosomal PD-L1 secreted by glioblastoma stem-like cells were identified to participate in $\mathrm{T}$ cell immunosuppression by inhibition of $\mathrm{CD} 4^{+}$and $\mathrm{CD} 8^{+}$ Tcell activation. ${ }^{74}$ PD-L1-containing exosomes isolated from NSCLC patients could inhibit the activity of CD8 ${ }^{+}$ $\mathrm{T}$ cells by reducing the production of interleukin 2 (IL-2) and IFN- $\gamma$ in a dose-dependent manner. Of note, exosomes with high levels of PD-L1 (PD-L1 ${ }^{\text {high }}$ exosomes) showed a strong potency to suppress the production of IL-2 and IFN- $\gamma$, whereas exosomes with lower levels of PD-L1 (PD-L1 ${ }^{\text {low }}$ exosomes) exerted no significant effect. PD-L1 ${ }^{\text {high }}$ exosomes could also significantly induce apoptosis in $\mathrm{CD}^{+} \mathrm{T}$ cells through PD-1/PD-L1 interaction. ${ }^{75}$ Furthermore, in patients with HNSCC, exosomal PD-L1 were able to suppress the proliferation of $\mathrm{CD} 4^{+} \mathrm{T}$ cells, induce apoptosis in $\mathrm{CD}^{+} \mathrm{T}$ cells, and enhance the suppressor activity of Treg cells, depending on the level of PD-L1 in exosomes. PD-L1 ${ }^{\text {high }}$ exosomes were found to be more effective in the suppression of T cells, compared with PD-L1 ${ }^{\text {low }}$ exosomes. ${ }^{71}$ An in vivo study revealed that exosomal PD-L1 isolated from murine (SCCVII) and human (SCC90) HNSCC cell lines could enhance tumor progression in a mouse model of human oral-oesophageal

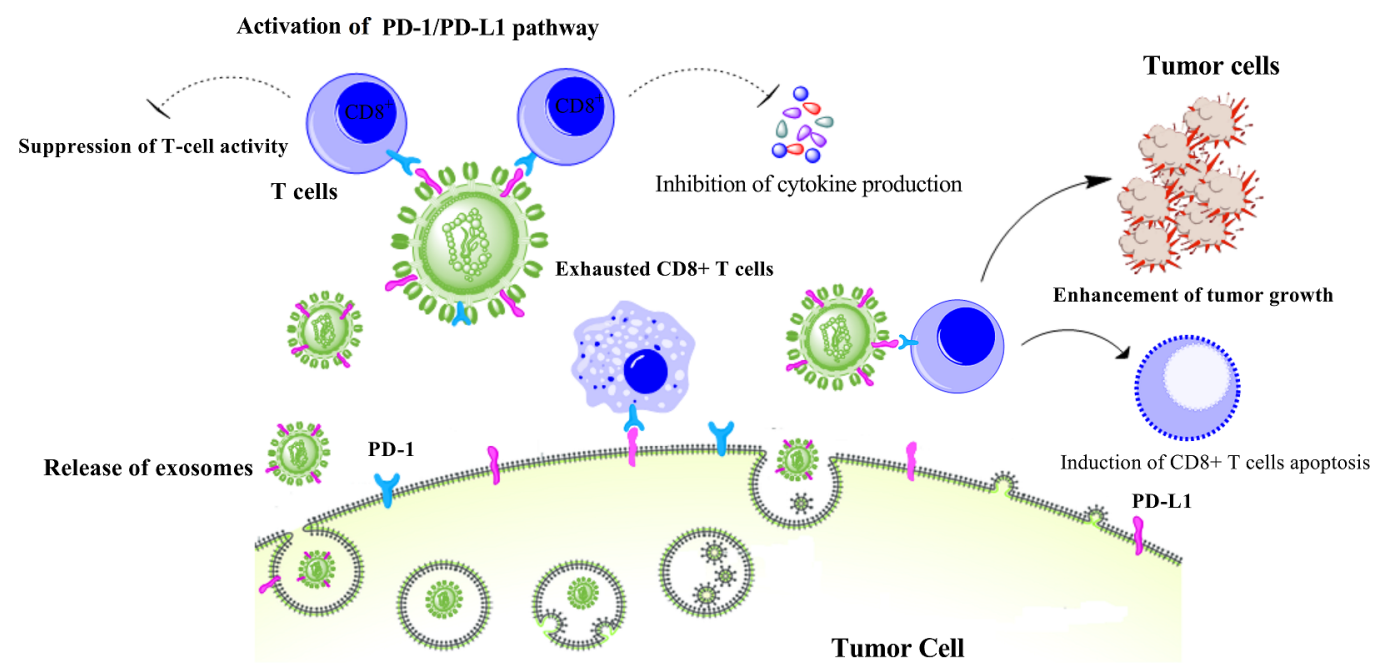

Figure 1 The role of exosomal PD-L1 in cancer progression. Tumor cells secrete exosomes containing PD-L1 leading to suppression of immunity by reducing T-cell activity and inhibition of interferon-gamma and interleukin 2 production as well as reducing total number of $\mathrm{CD}^{+} \mathrm{T}$ cells by inducing apoptosis through PD-1/PD-L1 pathway. PD-1, programmed cell death protein 1; PD-L1, programmed death-ligand 1. 
cancer through reducing migration of $\mathrm{CD} 4^{+}$and $\mathrm{CD} 8^{+} \mathrm{T}$ cells toward the tumor site. ${ }^{76}$ Further studies on a mouse model of prostate cancer exhibits that tumor-secreted exosomal PD-L1 can travel to the tumor's draining lymph nodes where it inhibits $\mathrm{T}$ cell activation and eventually leads to $\mathrm{T}$ cell exhaustion and reducing spleen size. ${ }^{73}$ In sum, such experimental and clinical studies strongly emphasis that exosomal PD-L1 through reprinting function of cell-surface PD-L1 can inhibit T cell immunity and enhance tumor growth in different tumor types, including prostate, head and neck, oral-oesophageal, and colorectal cancer.

\section{PD-L1 IN EXOSOMES AND THE ACQUIRED RESISTANCE TO ANTI-PD-1/PD-L1 THERAPY}

There is convincing preclinical and clinical evidence that shows the effective role of exosomal PD-L1 in the relatively low response rate of anti-PD-L1/PD-1 therapy. Clinical significance of plasma circulating exosomal PD-L1 has been indicated in patients with head and neck cancer, ${ }^{71} 77$ gastric cancer, ${ }^{7879}$ NSCLC, $^{72}$ pancreatic cancer, ${ }^{80}$ and melanoma. ${ }^{69}$ Among melanoma patients, the pretreatment levels of circulating exosomal PD-L1 were found to be negatively correlated with disease response to pembrolizumab. Importantly, a higher level of circulating exosomal PD-L1 before the treatment was associated with poorer clinical outcomes. ${ }^{69}$

There are also preclinical studies that indicate the removal of exosomal PD-L1 can increase the response rate of the anti-PD-L1 blockade, supporting the critical impact of exosomal PD-L1 in therapeutic resistance to anti-PD-L1 antibody treatment. In the mouse $4 \mathrm{~T} 1$ breast tumor model, it was shown that the elimination of exosomal PD-L1 by inhibiting exosome secretion using knockdown of Rab27a in tumor cells considerably improved the efficiency of anti-PD-1 therapy and suppressed the tumor growth. ${ }^{70}$ The TRAMP-C2 model is a syngeneic model of prostate cancer ${ }^{81}$ that, like human prostate cancer, is resistant to anti-PD-L1 blockade. ${ }^{82}$ Removal of tumor exosomal PD-L1 from TRAMP-C2 cells using genetic mutations could efficiently enhance the effect of PD-L1 blockade, which included inhibition of tumor growth, elevated cellularity of the spleen, and the activation of a $\mathrm{T}$ cell response in lymph nodes with similar effects on the various activation, exhaustion, and proliferation markers. Interestingly, the injection of in vitro collected exosomes carrying PD-L1 was found to reverse all these outcomes in the TRAMP-C2 model, whereas, in the absence of PD-L1, the effect of the exogenously introduced exosomes was very low. ${ }^{82}$ Similarly, the removal of exosomal PD-L1 in the colorectal MC38 model inhibited tumor growth and extended survival through PD-L1 blockade treatment. ${ }^{73}$ Mechanistically, it was suggested that high levels of exosomal PD-L1 might reflect the "exhaustion" of T cells to a stage at which they can no longer be reinvigorated by anti-PD-1 therapy. ${ }^{73}$
These findings are all consistent with exosomal PD-L1-mediated resistance to current anti-PD-L1/PD-1 therapies. However, the mechanism underlying the therapeutic resistance of exosomal PD-L1 is still unknown. One possible mechanism is that the delivered anti-PD-L1 antibodies can directly bind to exosomal PD-L1, and thereby few antibodies remain to inhibit PD-L1 on the surface of tumor cells. In addition, high levels of exosomal PD-L1 can compete with the administered anti-PD-L1/PD-1 antibodies (figure 2). Likewise, exosomes may reach targets that are sequestered from the antibody's effect. Therefore, combination therapy using anti-exosomal treatment may synergize the therapeutic efficiency of anti-PD-1/ PD-L1 blockade.

\section{EXOSOME ELIMINATION AS AN ADD-ON THERAPY ENHANCING POTENCY OF ANTI-PD-1/PD-L1 ANTIBODIES}

There is preclinical evidence that shows loss of PD-L1 expression from primary tumors along with the elimination of exosome secretion via pharmacological intervention or genetic manipulation mitigate the metastatic burden and elevate overall survival in a variety of tumorbearing mice ${ }^{697073}$ Combination treatment with GW4869, an inhibitor of exosome secretion, and anti-PD-L1 mAb demonstrated the highest reduction in primary tumor burden in mice bearing 4T-1 tumor, showing a synergistic association between immune checkpoint inhibitors and exosome elimination. ${ }^{70}$ Supporting this, anti-PD-L1 efficacy was markedly enhanced in mice harboring tetracycline-inducible Rab27 knockdown $4 \mathrm{~T} 1$ cells. $^{70}$ Of equal relevance, an Rab27 ${ }^{-/-}$MC38 cell line dramatically enlarged overall survival in mice receiving anti-PD-L1 mAb compared with either monotherapy alone ${ }^{73}$ Similar results were also observed by an independent group in other tumor models. ${ }^{69}$ Altogether, these findings suggest that exosome elimination may act as an effective add-on therapy to improve the therapeutic efficiency of antiPD-1/PD-L1 blockade in patients with cancer. Exosomes can be eliminated through suppressing their generation and secretion from tumor cells or removal of circulating exosomes from the bloodstream using haemofiltration.

\section{Pharmacological approaches for suppressing exosome generation and secretion}

Elimination of circulating exosomes has emerged as a novel and potentially useful therapeutic strategy for the development of anticancer drugs. ${ }^{83}$ Many reports have already shown that the reduction of exosome secretion or secreted exosomes, achieved by using a chemical inhibitor, ${ }^{84}$ genetic manipulation, ${ }^{86}$ or antibody, ${ }^{87}$ can suppress cancer metastasis and improve the efficiency of cancer chemotherapy. The effective strategies for exosome suppression are the use of pharmacological agents that reduce the level of exosomes by targeting different molecules involving in the generation, packaging, and release of them. However, since exosomes are implicated in intercellular communications and 

(1) Acquired resistance to
anti-PD1/PD-L1 therapy
(2) Exosome elimination enhancing

anti-PD1/PD-L1 antibodies
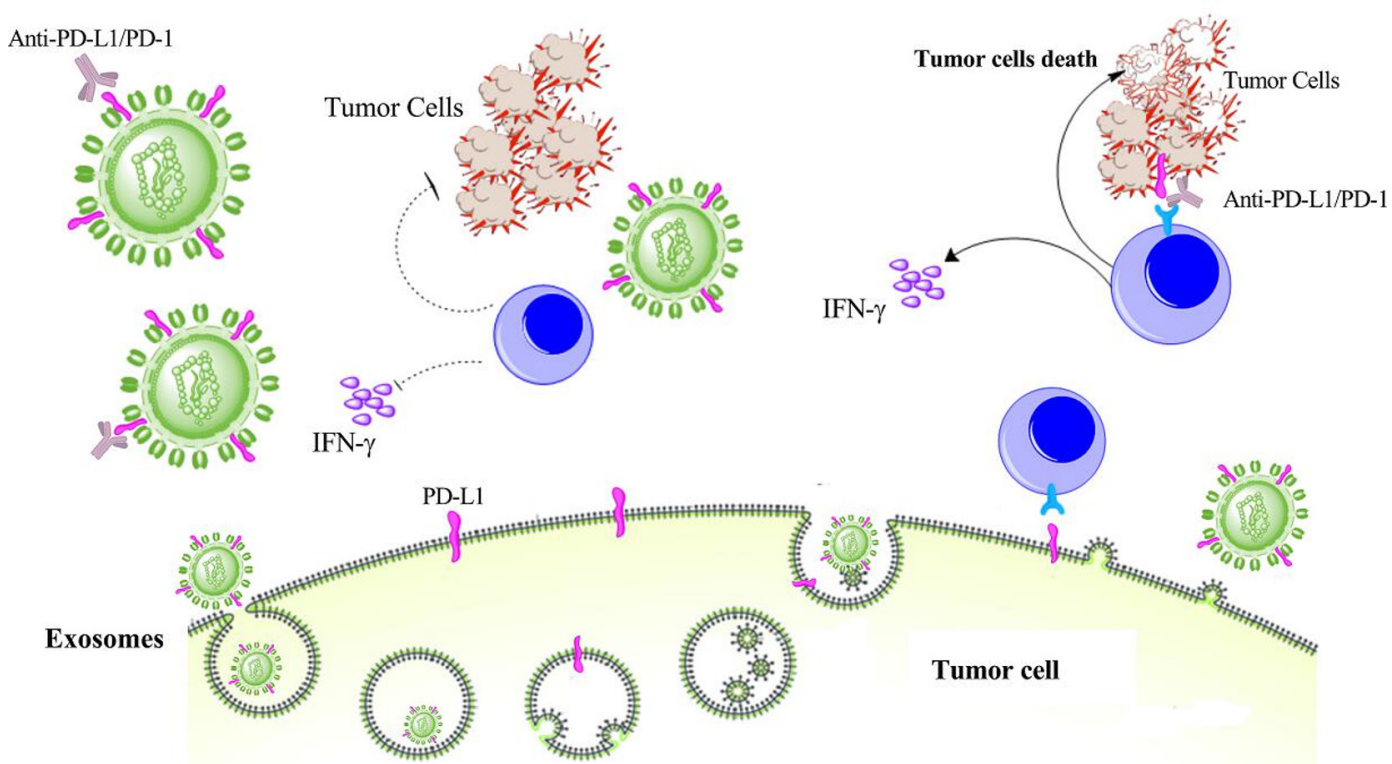

Figure 2 Exosomal PD-L1 induces acquired resistance to anti-PD-1/PD-L1 therapy. Exosomes carrying PD-L1 limit effectiveness of anti-PD-1/PD-L1 therapy through binding to antibodies and suppression of T-cell activity. However, elimination of PD-L1 exosomes can improve anti-PD-1/PD-L1 therapy. IFN- $\gamma$, interferon-gamma; PD-1, programmed cell death protein 1 ; PD-L1, programmed death-ligand 1.

maintaining normal cellular physiology, this represents the most important limitations to be used as a therapeutic strategy, due to the potential toxicity and other side-effects caused by any partial or temporary inhibition of exosome secretion from normal cells when a drug candidate inhibits the secretion of exosomes from cancer cells. Using exosome inhibitors developed by drug repurposing strategy, a process of finding new indications for existing FDA-approved drugs, can reduce concerns about safety/toxicity, because these agents have already passed toxicity and safety tests in humans. ${ }^{858}$ For example, FDAapproved drugs, dimethyl amiloride (DMA) and omeprazole reduce exosome secretion through targeting $\mathrm{H}^{+}$/ $\mathrm{Na}^{+}$and $\mathrm{Na}^{+} / \mathrm{Ca}^{+}$channels ${ }^{89}$ that are known to involve in exosome release. ${ }^{90}$ DMA has been found to reduce the production of exosomes and eliminate their immune suppressive effects in tumor-bearing hosts and enhance the antitumor efficacy of the cyclophosphamide as a chemotherapeutic drug. ${ }^{89}$ Another possible option for inhibiting exosome secretion is the use of proton pump inhibitors (PPIs), which are widely prescribed for mitigating gastric acid. ${ }^{91}$ Vacuolar $\mathrm{H}^{+}$-ATPases can regulate $\mathrm{pH}$ of extracellular microenvironments and involve in tumor progression. PPIs through inhibiting vacuolar $\mathrm{H}^{+}$ATPase-driven efflux pumps impair the release of acidic vesicles and vesicle-like structures by tumor cells and enhance the efficacy of chemotherapeutic agents. ${ }^{91}{ }^{92}$ In addition, ceramide is known to involve in biogenesis and release of exosomes, and sphingomyelinases, enzymes promoting ceramide synthesis, participate in sorting the cargo as well. Notably, cell treatment with sphingomyelinase inhibitors was found to markedly reduce the release of exosomes. ${ }^{93}$ Moreover, sulfisoxazole, an FDA-approved oral antibiotic, was also identified as an inhibitor of exosome secretion from breast cancer cells through interference with endothelin receptor A, a member of G protein-coupled receptor (GPCR) family that plays a critical role in the biogenesis and secretion of exosome in breast cancer cells. ${ }^{88}$ Of note, sulfisoxazole exhibited significant antitumor and anti-metastatic impacts in mouse models of breast cancer xenografts, the decreased expression of proteins participated in exosome biogenesis and secretion, and induced lysosomal degradation of multivesicular endosomes. ${ }^{88}$ Interestingly, a quantitative high throughput screen assay of many pharmacologically active compounds approved for clinical application uncovers the lead compounds tipifarnib, neticonazole, climbazole, ketoconazole, and triademenol that inhibit exosome biogenesis and/or release by aggressive prostate cancer calls. ${ }^{85}$ Hence, utility of drug-repurposing for preventing exosome secretion may provide a useful and safe approach for unmasking the inhibitory effects of exosomal PD-L1 on immune function and therapeutic responses in cancer.

\section{Exosome removal using extracorporeal haemofiltration}

Another alternative promising approach is exosome removal from the blood circulation by extracorporeal haemofiltration, which would not represent the possible drug toxicity or interactions, thereby suggesting an 
advantage over pharmacological strategies. Aethlon ADAPT (adaptive dialysis-like affinity platform technology) therapy is a therapeutic haemofiltration approach with a mechanism of action similar to kidney dialysis or continuous renal replacement therapy method. In this approach, plasma and blood cells pass through the hollow-fiber dialysis cartridges $(<200 \mathrm{~nm}$ pore) that are packed with the immobilized affinity matrix. The component of matrices can be customized with our purpose and can be composed of different affinity agents including monoclonal antibodies, aptamers, and various ligands to specifically capture and remove soluble proteins from the plasma. During the circulation of plasma over the cartridge, target molecules can selectively interact with the matrix and be isolated from other molecules, while unbound components and blood cells of serum pass through the device.

The safety and efficacy of the Aethlon haemopurifier device have been evaluated in clearing the hepatitis $\mathrm{C}$ virus (HCV) from the blood of HCV-positive end-stage renal disease patients by using lectin affinity plasmapheresis. ${ }^{94}$ Of note, ADAPT therapy can be also used for targeting and removal of exosomes as an adjuvant treatment in cancer therapy. Marleau et al showed that haemopurifier technique can be employed for capturing tumor-derived exosomes based on specific molecules on the exosomal surface, such as high mannose exosomes to remove by lectins or HER2 positive exosomes to remove with anti-HER2 antibodies. ${ }^{95}$ Hence, it can be proposed that PD-L1 positive exosomes can be eliminated by Aethlon haemopurifier device equipped with a matrix containing anti-PD-L1 antibody, suggesting an adjunct therapeutic candidate to the standard of care cancer treatments. However, considering the role of circulating exosomal PD-L1 on the mediation of systemic immunosuppression, there are concerns about possible severe irAEs along with their elimination. Therefore, although some preclinical studies showed improved survival with no significant adverse effect in exosomal PD-L1 depleted mice bearing tumors ${ }^{73}$ further preclinical toxicity testing is important to address the safety of exosomal PD-L1 elimination.

\section{CONCLUSION AND FUTURE PERSPECTIVES}

This review highlights and summarizes current knowledge regarding the role of exosomal PD-L1 in acquired resistance to anti-PD-1/PD-L1 therapy of cancer. In brief, exosomal PD-L1 can reprint the effect of tumor cellsurface PD-L1 in cancer progression by enabling tumor cells to escape from antitumor immunity. Exosomes can also deliver PD-L1 from origin tumor cells to other cell types with low or no PD-L1 expression and thereby suppress systemic antitumor immunity and memory. Notably, plasma levels of exosomal PD-L1 are negatively correlated with the rate of response to anti-PD-1/PD-L1 therapy in patients with cancer. Of note, there is evidence that indicates the removal of exosomal PD-L1 can increase the response to the anti-PD-L1 blockade in animal models. These findings are all consistent with exosomal PD-L1-mediated resistance to current anti-PD-L1/PD-1 therapies. Although the mechanism underlying therapeutic resistance of exosomal PD-L1 is still unknown, some possible mechanisms are proposed. Importantly, circulating exosomal PD-L1 can interact with anti-PD-1/ PD-L1 antibodies and limit their therapeutic efficiency. Moreover, high levels of exosomal PD-L1 can compete with the administered anti-PD-L1/PD-1 antibodies. It is also possible that exosomes attain targets that are sequestered from the antibody's effect. Therefore, combination therapy using anti-exosomal treatment may synergize the therapeutic efficiency of anti-PD-1/PD-L1 blockade. However, since normal cell-secreted exosomes are critical for maintaining normal physiology, a drug candidate should selectively inhibit cancer-specific exosomes to decrease potential toxicity/side-effects. As an alternative approach, removal of secreted PD-L1 ${ }^{+}$exosomes from the bloodstream may also be an efficient strategy, of course, if toxicity studies verify its safety and the lack of irAEs.

Correction notice This article has been corrected since it first published. The provenance and peer review statement has been included and Dr. MomtaziBorojeni's affiliation has been corrected.

Contributors $\mathrm{ZY}$ and SC contributed to the conception and design of the work. ZY and MY were major contributors in writing the manuscript. TM and CZ performed the database search and wrote a part of the manuscript. SH and MRK prepared the table and designed figures. AAM-B read and approved the final manuscript.

Funding This study was supported by the Science and Technology Program of Guangzhou (No. 201904010043), National Natural Science Foundation (No. 81802892, No. 82072637), and Medical Scientific Research Foundation of Guangdong (No. A2019453).

Competing interests None declared.

Patient consent for publication Not required.

Provenance and peer review Not commissioned; externally peer reviewed.

Open access This is an open access article distributed in accordance with the Creative Commons Attribution Non Commercial (CC BY-NC 4.0) license, which permits others to distribute, remix, adapt, build upon this work non-commercially, and license their derivative works on different terms, provided the original work is properly cited, appropriate credit is given, any changes made indicated, and the use is non-commercial. See http://creativecommons.org/licenses/by-nc/4.0/.

ORCID iD

Amir Abaas Momtazi-Borojeni http://orcid.org/0000-0002-4376-1083

\section{REFERENCES}

1 Wei SC, Duffy CR, Allison JP. Fundamental mechanisms of immune checkpoint blockade therapy. Cancer Discov 2018;8:1069-86.

2 Agata Y, Kawasaki A, Nishimura H, et al. Expression of the PD-1 antigen on the surface of stimulated mouse $\mathrm{T}$ and B lymphocytes. Int Immunol 1996;8:765-72.

3 Keir ME, Liang SC, Guleria I, et al. Tissue expression of PD-L1 mediates peripheral T cell tolerance. J Exp Med 2006;203:883-95.

4 Yokosuka T, Takamatsu M, Kobayashi-Imanishi W, et al. Programmed cell death 1 forms negative costimulatory microclusters that directly inhibit T cell receptor signaling by recruiting phosphatase SHP2. J Exp Med 2012;209:1201-17.

5 Garcia-Diaz A, Shin DS, Moreno BH, et al. Interferon receptor signaling pathways regulating PD-L1 and PD-L2 expression. Cell Rep 2017;19:1189-201.

$6 \mathrm{Wu}$ Y, Chen W, Xu ZP, et al. Pd-L1 distribution and perspective for cancer Immunotherapy-Blockade, knockdown, or inhibition. Front Immunol 2019;10:2022.

7 Sfanos KS, Bruno TC, Meeker AK, et al. Human prostate-infiltrating CD8+ T lymphocytes are oligoclonal and PD-1+. Prostate 2009;69:1694-703. 
8 Ahmadzadeh M, Johnson LA, Heemskerk B, et al. Tumor antigenspecific CD8 $\mathrm{T}$ cells infiltrating the tumor express high levels of $\mathrm{PD}-1$ and are functionally impaired. Blood 2009;114:1537-44.

9 Dong H, Strome SE, Salomao DR, et al. Tumor-Associated B7$\mathrm{H} 1$ promotes T-cell apoptosis: a potential mechanism of immune evasion. Nat Med 2002;8:793-800.

10 Blank C, Brown I, Peterson AC, et al. PD-L1/B7H-1 Inhibits the Effector Phase of Tumor Rejection by T Cell Receptor (TCR) Transgenic CD8 ${ }^{+}$T Cells. Cancer Res 2004;64:1140-5.

11 Iwai $Y$, Ishida M, Tanaka Y, et al. Involvement of PD-L1 on tumor cells in the escape from host immune system and tumor immunotherapy by PD-L1 blockade. Proc Natl Acad Sci U S A 2002;99:12293-7.

12 Rittmeyer A, Barlesi F, Waterkamp D, et al. Atezolizumab versus docetaxel in patients with previously treated non-small-cell lung cancer (oak): a phase 3, open-label, multicentre randomised controlled trial. Lancet 2017;389:255-65.

13 Baxi S, Yang A, Gennarelli RL, et al. Immune-Related adverse events for anti-PD-1 and anti-PD-L1 drugs: systematic review and metaanalysis. BMJ 2018;360:k793.

14 Friedman CF, Proverbs-Singh TA, Postow MA. Treatment of the immune-related adverse effects of immune checkpoint inhibitors. JAMA Oncology 2016;2:1346-53.

15 Kwok G, Yau TCC, Chiu JW, et al. Pembrolizumab (Keytruda). Hum Vaccin Immunother 2016;12:2777-89.

16 Peters S, Kerr KM, Stahel R. Pd-1 blockade in advanced NSCLC: a focus on pembrolizumab. Cancer Treat Rev 2018;62:39-49.

17 Sim F, Leidner R, Bell RB. Immunotherapy for head and neck cancer. Oral Maxillofac Surg Clin North Am 2019;31:85-100.

18 Bellmunt J, de Wit R, Vaughn DJ, et al. Pembrolizumab as secondline therapy for advanced urothelial carcinoma. N Engl J Med Overseas Ed 2017;376:1015-26.

19 Herbst RS, Soria J-C, Kowanetz M, et al. Predictive correlates of response to the anti-PD-L1 antibody MPDL3280A in cancer patients. Nature 2014;515:563-7.

20 Tumeh PC, Harview CL, Yearley JH, et al. Pd-1 blockade induces responses by inhibiting adaptive immune resistance. Nature 2014;515:568-71.

21 Im SJ, Hashimoto M, Gerner MY, et al. Defining CD8+ T cells that provide the proliferative burst after PD-1 therapy. Nature 2016;537:417-21.

22 Huang AC, Postow MA, Orlowski RJ, et al. T-Cell invigoration to tumour burden ratio associated with anti-PD-1 response. Nature 2017:545:60-5.

23 Forde PM, Chaft JE, Smith KN, et al. Neoadjuvant PD-1 blockade in resectable lung cancer. N Engl J Med Overseas Ed 2018;378:1976-86.

24 Bengsch B, Johnson AL, Kurachi M, et al. Bioenergetic Insufficiencies due to metabolic alterations regulated by the inhibitory receptor PD-1 are an early driver of CD8 + T cell exhaustion. Immunity 2016;45:358-73.

25 Gubin MM, Zhang X, Schuster H, et al. Checkpoint blockade cancer immunotherapy targets tumour-specific mutant antigens. Nature 2014:515:577-81.

26 Loke P'ng, Allison JP. Pd-L1 and PD-L2 are differentially regulated by Th1 and Th2 cells. Proc Natl Acad Sci U S A 2003;100:5336-41.

27 Dahan R, Sega E, Engelhardt J, et al. FcyRs modulate the anti-tumor activity of antibodies targeting the PD-1/PD-L1 axis. Cancer Cell 2015:28:285-95.

28 Jenkins RW, Barbie DA, Flaherty KT. Mechanisms of resistance to immune checkpoint inhibitors. Br J Cancer 2018;118:9-16.

29 Polk A, Svane I-M, Andersson M, et al. Checkpoint inhibitors in breast cancer - current status. Cancer Treat Rev 2018;63:122-34.

30 Sheng Z, Zhu X, Sun Y, et al. The efficacy of anti-PD-1/PD-L1 therapy and its comparison with EGFR-TKls for advanced non-smallcell lung cancer. Oncotarget 2017;8:57826-35.

31 Ribas A. Adaptive immune resistance: how cancer protects from immune attack. Cancer Discov 2015;5:915-9.

32 Reck M, Rodríguez-Abreu D, Robinson AG, et al. Pembrolizumab versus chemotherapy for PD-L1-positive non-small-cell lung cancer. N Engl J Med Overseas Ed 2016;375:1823-33.

33 Ding W, LaPlant BR, Call TG, et al. Pembrolizumab in patients with CLL and Richter transformation or with relapsed CLL. Blood 2017;129:3419-27.

34 Patel SP, Kurzrock R. Pd-L1 expression as a predictive biomarker in cancer immunotherapy. Mol Cancer Ther 2015;14:847-56.

35 Yagi T, Baba Y, Ishimoto T, et al. Pd-L1 expression, tumor-infiltrating lymphocytes, and clinical outcome in patients with surgically resected esophageal cancer. Ann Surg 2019;269:471-8.

36 Teng MWL, Ngiow SF, Ribas A, et al. Classifying cancers based on T-cell infiltration and PD-L1. Cancer Res 2015;75:2139-45.
37 Thommen DS, Koelzer VH, Herzig P, et al. A transcriptionally and functionally distinct $\mathrm{PD}-1^{+} \mathrm{CD} 8^{+} \mathrm{T}$ cell pool with predictive potential in non-small-cell lung cancer treated with PD-1 blockade. Nat Med 2018;24:994-1004

38 Sade-Feldman M, Yizhak K, Bjorgaard SL, et al. Defining T cell states associated with response to checkpoint immunotherapy in melanoma. Cell 2018;175:e1020:998-1013.

39 Tran E, Ahmadzadeh M, Lu Y-C, et al. Immunogenicity of somatic mutations in human gastrointestinal cancers. Science 2015:350:1387-90.

40 Tran E, Turcotte S, Gros A, et al. Cancer immunotherapy based on mutation-specific CD4+ T cells in a patient with epithelial cancer. Science 2014;344:641-5.

41 Monach PA, Meredith SC, T.Siegel C, et al. A unique tumor antigen produced by a single amino acid substitution. Immunity 1995;2:45-59.

42 Robbins PF, El-Gamil M, Li YF, et al. A mutated beta-catenin gene encodes a melanoma-specific antigen recognized by tumor infiltrating lymphocytes. J Exp Med 1996;183:1185-92.

43 Dubey P, Hendrickson RC, Meredith SC, et al. The immunodominant antigen of an ultraviolet-induced regressor tumor is generated by a somatic point mutation in the DEAD box helicase p68. $J$ Exp Med 1997; 185:695-706.

44 Lennerz V, Fatho M, Gentilini C, et al. The response of autologous T cells to a human melanoma is dominated by mutated neoantigens. Proc Natl Acad Sci U S A 2005;102:16013-8.

45 Rizvi NA, Hellmann MD, Snyder A, et al. Mutational landscape determines sensitivity to PD-1 blockade in non-small cell lung cancer. Science 2015;348:124-8.

46 Chen Y, Liu Q, Chen Z, et al. Pd-L1 expression and tumor mutational burden status for prediction of response to chemotherapy and targeted therapy in non-small cell lung cancer. J Exp Clin Cancer Res 2019;38:193.

47 Hellmann MD, Nathanson T, Rizvi H, et al. Genomic features of response to combination immunotherapy in patients with advanced non-small-cell lung cancer. Cancer Cell 2018;33:e844:843-52.

48 Hellmann MD, Ciuleanu T-E, Pluzanski A, et al. Nivolumab plus ipilimumab in lung cancer with a high tumor mutational burden. $N$ Engl J Med 2018;378:2093-104.

49 Rosenberg JE, Hoffman-Censits J, Powles T, et al. Atezolizumab in patients with locally advanced and metastatic urothelial carcinoma who have progressed following treatment with platinum-based chemotherapy: a single-arm, multicentre, phase 2 trial. The Lancet 2016;387:1909-20.

50 Riaz N, Havel JJ, Makarov V, et al. Tumor and microenvironment evolution during immunotherapy with nivolumab. Cell 2017;171:e916:934-49.

51 Hugo W, Zaretsky JM, Sun L, et al. Genomic and transcriptomic features of response to anti-PD-1 therapy in metastatic melanoma. Cell 2016;165:35-44.

52 Goodman AM, Kato S, Bazhenova L, et al. Tumor mutational burden as an independent predictor of response to immunotherapy in diverse cancers. Mol Cancer Ther 2017;16:2598-608.

53 Legrand FA, Gandara DR, Mariathasan S, et al. Association of high tissue TMB and atezolizumab efficacy across multiple tumor types. JCO 2018;36:12000.

54 Yarchoan M, Hopkins A, Jaffee EM. Tumor mutational burden and response rate to PD-1 inhibition. N Engl J Med Overseas Ed 2017;377:2500-1.

55 Le DT, Durham JN, Smith KN, et al. Mismatch repair deficiency predicts response of solid tumors to PD-1 blockade. Science 2017:357:409-13.

56 Le DT, Uram JN, Wang $\mathrm{H}$, et al. Pd-1 blockade in tumors with mismatch-repair deficiency. N Engl J Med 2015;372:2509-20.

57 Germano G, Lamba S, Rospo G, et al. Inactivation of DNA repair triggers neoantigen generation and impairs tumour growth. Nature 2017:552:116-20.

58 Yi M, Jiao D, Xu H, et al. Biomarkers for predicting efficacy of PD-1/ PD-L1 inhibitors. Mol Cancer 2018;17:1-14.

59 Kalluri R. The biology and function of exosomes in cancer. $J$ Clin Invest 2016;126:1208-15.

60 Zhang L, Yu D. Exosomes in cancer development, metastasis, and immunity. Biochim Biophys Acta Rev Cancer 2019;1871:455-68.

61 Tavasolian F, Moghaddam AS, Rohani F. Exosomes: Effectual players in rheumatoid arthritis, 2020.

62 Moghaddam AS, Afshari JT, Esmaeili S-A, et al. Cardioprotective microRNAs: lessons from stem cell-derived exosomal microRNAs to treat cardiovascular disease. Atherosclerosis 2019;285:1-9.

63 Moghiman T, Barghchi B, Esmaeili S-A, et al. Therapeutic angiogenesis with exosomal microRNAs: an effectual approach for the treatment of myocardial ischemia, 2020: 1-9. 
64 Zhou R, Wang L, Zhao G. Circulating exosomal microRNAs as emerging non-invasive clinical biomarkers in heart failure: Mega bioroles of a nano bio-particle;72.

65 Greening DW, Gopal SK, Xu R, et al. Exosomes and their roles in immune regulation and cancer. Semin Cell Dev Biol 2015;40:72-81.

66 Yi M, Xu L, Jiao Y, et al. The role of cancer-derived microRNAs in cancer immune escape. J Hematol Oncol 2020;13:1-14.

67 Sun Z, Shi K, Yang S, et al. Effect of exosomal miRNA on cancer biology and clinical applications. Mol Cancer 2018;17:147.

68 McAndrews KM, Kalluri R. Mechanisms associated with biogenesis of exosomes in cancer. Mol Cancer 2019;18:52.

69 Chen G, Huang AC, Zhang W, et al. Exosomal PD-L1 contributes to immunosuppression and is associated with anti-PD-1 response. Nature 2018;560:382-6.

70 Yang Y, Li C-W, Chan L-C, et al. Exosomal PD-L1 harbors active defense function to suppress $T$ cell killing of breast cancer cells and promote tumor growth. Cell Res 2018;28:862-4.

71 Theodoraki M-N, Yerneni SS, Hoffmann TK, et al. Clinical Significance of PD-L1 $1^{+}$Exosomes in Plasma of Head and Neck Cancer Patients. Clin Cancer Res 2018;24:896-905.

$72 \mathrm{Li} \mathrm{C}$, Li C, Zhi C, et al. Clinical significance of PD-L1 expression in serum-derived exosomes in NSCLC patients. J Trans/ Med 2019;17.

73 Poggio M, Hu T, Pai C-C, et al. Suppression of exosomal PDL1 induces systemic anti-tumor immunity and memory. Cell 2019;177:e413:414-27.

74 Ricklefs FL, Alayo Q, Krenzlin H, et al. Immune evasion mediated by PD-L1 on glioblastoma-derived extracellular vesicles. Sci Adv 2018;4:eaar2766.

$75 \mathrm{Kim} \mathrm{DH}$, Kim H, Choi YJ, et al. Exosomal PD-L1 promotes tumor growth through immune escape in non-small cell lung cancer. Exp Mol Med 2019;51:1-13.

76 Razzo BM, Ludwig N, Hong C-S, et al. Tumor-Derived exosomes promote carcinogenesis of murine oral squamous cell carcinoma. Carcinogenesis 2020;41:625-33.

77 Theodoraki M-N, Yerneni S, Gooding WE, et al. Circulating exosomes measure responses to therapy in head and neck cancer patients treated with cetuximab, ipilimumab, and IMRT. Oncoimmunology 2019;8:e1593805.

78 Fan Y, Liu Y, Qu X. ASO author reflections: the prognostic role of exosomal PD-L1 in patients with gastric cancer. Ann Surg Oncol 2019;26:851-2.

79 Fan $\mathrm{Y}, \mathrm{Che} \mathrm{X}, \mathrm{Qu} \mathrm{J}$, et al. Exosomal PD-L1 retains immunosuppressive activity and is associated with gastric cancer prognosis. Ann Surg Oncol 2019;26:3745-55.

80 Lux A, Kahlert C, Grützmann R, et al. C-Met and PD-L1 on circulating exosomes as diagnostic and prognostic markers for pancreatic cancer. Int J Mol Sci 2019;20:3305.
81 Foster BA, Gingrich JR, Kwon ED, et al. Characterization of prostatic epithelial cell lines derived from transgenic adenocarcinoma of the mouse prostate (TRAMP) model. Cancer Res 1997;57:3325-30.

82 Yu P, Steel JC, Zhang M, et al. Simultaneous inhibition of two regulatory T-cell subsets enhanced interleukin-15 efficacy in a prostate tumor model. Proc Natl Acad Sci U S A 2012;109:6187-92.

83 EL Andaloussi S, Mäger I, Breakefield XO, et al. Extracellular vesicles: biology and emerging therapeutic opportunities. Nat Rev Drug Discov 2013;12:347-57.

84 Datta A, Kim H, Lal M, et al. Manumycin a suppresses exosome biogenesis and secretion via targeted inhibition of Ras/Raf/ERK1/2 signaling and hnRNP H1 in castration-resistant prostate cancer cells. Cancer Lett 2017;408:73-81.

85 Datta A, Kim H, McGee L, et al. High-Throughput screening identified selective inhibitors of exosome biogenesis and secretion: a drug repurposing strategy for advanced cancer. Sci Rep 2018;8:8161.

86 Bobrie A, Krumeich S, Reyal F, et al. Rab27A supports exosomedependent and -independent mechanisms that modify the tumor microenvironment and can promote tumor progression. Cancer Res 2012;72:4920-30.

87 Nishida-Aoki N, Tominaga N, Takeshita F, et al. Disruption of circulating extracellular vesicles as a novel therapeutic strategy against cancer metastasis. Mol Ther 2017;25:181-91.

$88 \mathrm{Im}$ E-J, Lee C-H, Moon P-G, et al. Sulfisoxazole inhibits the secretion of small extracellular vesicles by targeting the endothelin receptor $a$. Nat Commun 2019;10:1387.

89 Chalmin F, Ladoire S, Mignot G, et al. Membrane-Associated HSP72 from tumor-derived exosomes mediates STAT3-dependent immunosuppressive function of mouse and human myeloid-derived suppressor cells. J Clin Invest 2010;67:457-71.

90 Savina A, Furlán M, Vidal M, et al. Exosome release is regulated by a calcium-dependent mechanism in K562 cells. J Biol Chem 2003;278:20083-90.

91 Spugnini EP, Citro G, Fais S. Proton pump inhibitors as anti vacuolarATPases drugs: a novel anticancer strategy. J Exp Clin Cancer Res 2010;29:44.

92 Luciani F, Spada M, De Milito A, et al. Effect of proton pump inhibitor pretreatment on resistance of solid tumors to cytotoxic drugs. $J$ Natl Cancer Inst 2004;96:1702-13.

93 Trajkovic K, Hsu C, Chiantia S, et al. Ceramide triggers budding of exosome vesicles into multivesicular endosomes. Science 2008;319:1244-7.

94 Tullis RH, Duffin RP, Handley HH, et al. Reduction of hepatitis $\mathrm{C}$ virus using lectin affinity plasmapheresis in dialysis patients. Blood Purif 2009;27:64-9.

95 Marleau AM, Chen C-S, Joyce JA, et al. Exosome removal as a therapeutic adjuvant in cancer. $J$ Trans/ Med 2012;10:134. 
Correction: Mechanisms underlying low-clinical responses to PD-1/PD-L1 blocking antibodies in immunotherapy of cancer: a key role of exosomal $\mathrm{PD}-\mathrm{L} 1$

Yin Z, Yu M, Ma T, et al. Mechanisms underlying low-clinical responses to PD-1/PDL1 blocking antibodies in immunotherapy of cancer: a key role of exosomal PD-L1. J ImmunoTher Cancer 2021;9:e001698. doi: 10.1136/jitc-2020-001698

This article has been corrected since it was first published. Amir Abaas MomtaziBorojeni's affiliation has been updated to 'Department of Medical Biotechnology, School of Medicine, Alborz University of Medical Sciences, Karaj, Iran'.

Open access This is an open access article distributed in accordance with the Creative Commons Attribution Non Commercial (CC BY-NC 4.0) license, which permits others to distribute, remix, adapt, build upon this work non-commercially, and license their derivative works on different terms, provided the original work is properly cited, appropriate credit is given, any changes made indicated, and the use is non-commercial. See http://creativecommons.org/licenses/by-nc/4.0/.

(c) Author(s) (or their employer(s)) 2022. Re-use permitted under CC BY-NC. No commercial re-use. See rights and permissions. Published by BMJ.

J Immunother Cancer 2022;10:e001698corr2. doi:10.1136/jitc-2020-001698corr2

A) Check for updates 